\title{
Consistency in aggregation, quasilinear means and index numbers
}

\author{
Heikki Pursiainen \\ University of Helsinki and HECER
}

Discussion Paper No. 244

November 2008

ISSN 1795-0562

HECER - Helsinki Center of Economic Research, P.O. Box 17 (Arkadiankatu 7), FI-00014 University of Helsinki, FINLAND, Tel +358-9-191-28780, Fax +358-9-191-28781,

E-mail info-hecer@helsinki.fi, Internet www.hecer.fi 


\title{
Consistency in aggregation, quasilinear means and index numbers*
}

\begin{abstract}
In the calculation of economic aggregates it is often necessary to compute the value of these aggregates in some relevant subgroups as well as for the whole data. A method of calculation is said to be consistent in aggregation if it gives the same result regardless of whether it is applied directly to the whole data or to subaggregates calculated using the same method. The property has been well studied, yet a satisfactory general definition has been lacking. In this paper a definition of consistency in aggregation is given for a general class of aggregation rules. We show that any aggregation method satisfying this definition has a representation based on a commutative semigroup operation. Also, as an application we use algebraic methods to derive certain properties of index numbers satisfying consistency in aggregation. In particular, we show that any such index number formula is in fact a generalized weighted mean.
\end{abstract}

JEL Classification: C43.

Keywords: Aggregation, index number theory, algebra, linear algebra, semigroups.

Heikki Pursiainen

Department of Economics

University of Helsinki

P.O. Box 17

FI-00014 University of Helsinki

FINLAND

e-mail: heikki.pursiainen@helsinki.fi

*This is based on chapters 2-4 of my 2005 dissertation titled "Consistent Aggregation Methods and Index Number Theory". 


\section{Introduction}

The construction and use of aggregates, such as price indices, is one of the basic necessities of economic theory and practice. More often than not, an aggregate must be constructed not only for some population of interest but also for each of a number of relevant subsets of the population. From the point of view of economic analysis as well as computational economy, it would clearly be desirable to have the overall aggregate depend on only the subaggregates. To put this in more concrete terms, we would like to be able to calculate the aggregate in two or more stages in such a way that the result of each stage depend only on the one immediately preceding it. Two-stage aggregation and related issues have been studied in various branches of economics, but the most concrete formulation of the problem is perhaps found in the field of index number theory. Studies on two-stage consistency properties of index numbers include Vartia (1976) Diewert (1978), Blackorby and Primont (1980), Stuvel (1989), and Balk (1996). The relevant index number literature mostly considers a somewhat strengthened form of two-stage consistency, called consistency in aggregation. In addition to the condition that the two-stage procedure be consistent, it is required that (a) the overall aggregate can be derived from the subaggregates using the same aggregation method or functional form that was used to calculate the subaggregates in the first place and (b) that this is possible for any partition of the population.

While the intuition behind these requirements is clear enough, a precise yet general formulation of consistency in aggregation has proved to be surprisingly difficult to produce. Blackorby and Primont (1980) essentially solve the problem for the onevariable case, but do not have much to say on aggregation involving many variables. Gorman (1986) shows that given some additional conditions two-stage consistency leads to a quasilinear functional form (see below for a formal definition) for an index number formula, but does not specifically address the problem of defining consistency in aggregation. Balk (1996) proposes the quasilinear form as definition of consistency in aggregation. This definition is problematic, because some aggregation 
rules, such as taking a minimum or maximum are clearly consistent in aggregation, but fail to be quasilinear. Also, the definition is specific to aggregation of real or complex numbers, while there is nothing in the verbal characterization of consistency in aggregation that would necessitate such a restriction. We would therefore maintain that quasilinearity is a special case of consistency in aggregation rather than a definition of the property and that indeed that a satisfactory general definition has not been presented previously.

In the present study we propose such a general statement of consistency in aggregation for a large class of aggregation rules. An aggregation rule is defined simply to be any rule that attaches to any finite collection of elements of a set some unique element of that same set. This enables us to first to formulate consistency in aggregation without imposing any other a priori restrictions on the functional form of the aggregation rule. As was already mentioned, such a general definition has not to our knowledge existed until now. Second, we prove a representation theorem giving the implications of consistency in aggregation for the possible functional form of an aggregation rule. The theorem states that an aggregation rule satisfies consistency in aggregation if and only if it has a commutative semigroup representation. To put this result in more concrete terms, an aggregation rule satisfies consistency in aggregation if and only if it can be constructed by repeatedly applying a commutative and associative binary operation. The notion of consistency in aggregation is thus reduced to the concept of a commutative semigroup, a basic mathematical structure for which a well-developed theory exists. While of interest in itself, this new result provides a natural approach for analyzing the functional form of aggregation rules.

The semigroup representation result follows almost trivially from our definition of consistency in aggregation, but this is exactly the point. That the result seems almost self-evident given a clear formulation of the property shows that the source of confusion has indeed been the lack of such a formulation.

To illustrate the usefulness of our main results, we apply semigroup techniques to the index number problem. We describe how to represent index number formulas that are consistent in aggregation as semigroup operations and show that under loose 
conditions this implies a simple quasilinear functional form. Quasilinearity is shown using an extension of the classical results of Kolmogorov (1930) and Nagumo (1930) concerning quasilinearity of weighted means that we are able to derive using the semigroup property.

We have tried to make this article as self-contained as possible. As the mathematical concepts that are relevant to our study are not among the most widely used in economic literature, in Section 2 we give a brief explanation of these and establish the notation used. Also, in the section concerning index numbers, a brief outline of the index number problem is presented. Otherwise the article is organized as follows. In Section 3 we define an aggregation rule and consistency in aggregation. In Section 4 the semigroup representation theorem for aggregation rules satisfying consistency in aggregation is presented. In the Section 5 we give some examples and present an extension of the Kolmogorov-Nagumo quasilinearity results for weighted means. Finally, in Section 6 we discuss at some length the implications of consistency in aggregation for index number formulas. The quasilinearity theorem of the previous section is used to provide conditions under which an index number pair may be represented as a two-dimensional quasilinear mean.

\section{Basic concepts and notation}

Let $S$ be a set and $F: S^{2} \rightarrow S$ a function. Such a function is called a binary operation on $S$. To minimize the potential for confusion, we always use the multiplicative notation $F(x, y)=x y$ or $F(x, y)=x \circ y$ for such binary operations except for real addition for which we use the conventional notation.. If for any $x, y, z$ in $S$, $F(x, F(y, z))=F(F(x, y), z)$ or equivalently $x(y z)=(x y) z$ then the binary operation is associative. A set $S$ paired with an associative binary operation is called a semigroup. When there is no risk of confusion we often refer to the semigroup as simply $S$. If the binary operation of a semigroup is commutative, so that for any $x$ and $y$ in $S$ we have $x y=y x$, the semigroup is called a commutative (or Abelian) semigroup. 
If in a commutative semigroup the equation $a x=a y$ always implies $x=y$ the semigroup is a commutative cancellation semigroup. If $S$ and $S^{\prime}$ are semigroups and $f: S \rightarrow S^{\prime}$ satisfies $f(x y)=f(x) f(y)$ then $f$ is a homomorphism. If $f$ is a bijection then it is a (semigroup) isomorphism and $S$ and $S^{\prime}$ are isomorphic. Note that if $f: S \rightarrow R$ is any bijective function we may always define a semigroup on $R$ by setting $r s=f\left(f^{-1}(r) f^{-1}(s)\right)$ for $r, s \in R$. Clearly this semigroup is isomorphic with $S$ with $f$ an isomorphism. As a multiplicative notation is used for the semigroup operation it is also natural to use the exponential notation $x^{n}=\underbrace{x \cdots x}_{n \text { times }}$ as shorthand for repetition of the semigroup operation.

Let $R$ be a binary relation on the semigroup $S$. If $x R x^{\prime}$ and $y R y^{\prime}$ imply that $x y R x^{\prime} y^{\prime}$ then $S$ is a congruence. The set of equivalence classes of a congruence $R$, denoted $S \mid R$ is also a semigroup with respect to the operation that maps the equivalence classes of $x$ and $y$ to the equivalence class of $x y$.

A commutative semigroup with an identity element $e$ such that for all $x, e x=x$ is a commutative monoid. The identity element is always unique. If for any $x$ in a commutative monoid there exists $y$ such that $x y=e$ then the monoid is a commutative group. The element $y$ is denoted $y=x^{-1}$ and is called the inverse element of $x$. A semigroup $S$ is called topological when $S$ is a topological space and the semigroup operation is continuous with respect to the product topology of $S \times S$. A group is a topological group when it is a topological semigroup and inversion is also continuous. Two topological semigroups (groups) are isomorphic if there exists an isomorphism which is also a homeomorphism between the two semigroups (groups).

Let $\mathbb{R}_{++}$be the set of strictly positive reals, let $S$ be a semigroup with an operation $h: \mathbb{R}_{++} \times S$ denoted $h(\alpha, x)=x^{\alpha}$ which has the following properties for any $x, y$ in $S$ and $\alpha, \beta>0$ : (a) $\left(x^{\alpha}\right)^{\beta}=x^{\alpha \beta}$, (b) $(x y)^{\alpha}=x^{\alpha} y^{\alpha}$ and (c) $x^{\alpha} x^{\beta}=x^{\alpha+\beta}$. Then we call $S$ a cone semigroup. The intuition is that when the semigroup operation is interpreted as vector addition and the operation $x^{\alpha}$ as scalar multiplication then $S$ has similar properties as a convex cone in a linear space. If $S$ is a topological semigroup and $x^{\alpha}$ continuous in the relevant product topology then $S$ is called a topological cone semigroup. 


\section{Aggregation rules and consistency in aggrega- tion}

Consider an arbitrary set $X$ and call it a measurement scale. The aggregation problem we are concerned with is the aggregation of an arbitrary but finite number of measurements on the scale $X$ into an "aggregate measurement" on the same scale. That is, given any sequence $\left(x_{1}, \ldots, x_{n}\right) \in X^{n}$ we want to map it to an aggregate $\bar{x} \in X$ which is representative of this sequence in some sense. An aggregation rule on the measurement scale $X$ is simply a rule that tells us how to do this for any finite sequence of measurements. Formally, we define an aggregation rule $f$ to be a sequence of functions

$$
\left(f_{n}\right)_{n \in \mathbb{N}}, f_{n}: X^{n} \rightarrow X
$$

each member $f_{n}$ of which specifies how any sequence of $n$ measurements is to be aggregated. The function $f_{1}$ is included for completeness, and we adopt the convention that it is the identity mapping of $X$. Thus, given a sequence of measurements, $\left(x_{1}, \ldots, x_{n}\right) \in X^{n}$, say, the aggregation rule $f$ assigns a unique aggregate $\bar{x}=f_{n}\left(x_{1}, \ldots, x_{n}\right)$ to it. The formulation avoids any problems related to the meaning of the same rule or method in different situations. Essentially, the problem is circumnavigated by making it a matter of arbitrary decision what to call the same rule. Applying the same rule for a sequence of length $n$ and one with length $m$ simply means applying functions $f_{n}$ and $f_{m}$ respectively.

A point that perhaps needs to be briefly addressed is that we have restricted attention to aggregation rules that map measurements on scale $X$ to the same scale. This is indeed a restriction, but a less severe one as one might think at first. Below we will show that properly formulated, many if not most typical aggregation rules, e.g. different means and index number formulas fall into this category. The basic idea of how this is done is that the dimensionality of the measurement scale is increased by including auxiliary information such as data on a weighting variable explicitly in 
the measurements. The rationale for working with such representations is simple. As the aggregate is on the same scale as the original measurements, it is possible to use subaggregates directly as data for the aggregation rule. This makes it easy to formulate the two-step procedure that is central to the concept of consistency in aggregation.

A permutation $\left(x_{1}^{\prime}, \ldots, x_{n}^{\prime}\right)$ of a sequence of measurements $\left(x_{1}, \ldots, x_{n}\right)$ is obtained by putting $x_{i}^{\prime}=x_{j(i)}$, where $j$ is any bijection from the set $\{1, \ldots, n\}$ to itself. It is also always possible to partition a sequence into $K$ non-empty subsequences $\left(x_{k, 1}, \ldots, x_{k, n}\right)$, with $k=1, \ldots, K, 1 \leq K \leq n$ and $n_{1}+\ldots+n_{K}=n$.

An aggregation rule $f$ is said to be consistent in aggregation (CIA) if and only if it satisfies the following equality

$$
f_{K}\left(f_{n_{1}}\left(x_{1,1}^{\prime}, \ldots, x_{1, n_{1}}^{\prime}\right), \ldots, f_{n_{K}}\left(x_{K, 1}^{\prime}, \ldots, x_{K, n_{K}}^{\prime}\right)\right)=f_{n}\left(x_{1}, \ldots x_{n}\right)
$$

for any partition of any permutation $\left(x_{1}^{\prime}, \ldots, x_{n}^{\prime}\right)$ of any sequence $\left(x_{1}, \ldots, x_{n}\right)$.

The correspondence to the verbal characterization discussed is straightforward. First, the same-scale property of aggregation rules means that the two-step consistency requirement outlined in the introduction can be expressed simply by requiring that the total aggregate be a function of the subaggregates only. The two-stage aggregation is carried out by different functions in the aggregation rule $f$, which corresponds to the requirement (a) above, or that the overall aggregate and the subaggregates be calculated using the same method. Finally, property (b) is taken care of by requiring that equation (CIA) hold for any partitions and permutations of the measurements.

\section{A semigroup representation theorem}

While the definition of consistency in aggregation given in the previous section is straightforward enough, it is somewhat cumbersome. In fact consistency in aggregation is equivalent to the existence of a very simple representation for the aggregation 
rule, given in the next theorem.

Theorem 1 An aggregation rule is CIA if and only if it has the commutative semigroup representation:

$$
\begin{aligned}
f_{1}\left(x_{1}\right) & =x_{1} \\
f_{n}\left(x_{1}, \ldots, x_{n}\right) & =x_{1} \cdots x_{n} .
\end{aligned}
$$

Proof. Let $f$ be CIA. Note first that CIA implies that the following recursive algorithm may be used to define $f_{n+1}$ using $f_{n}$ :

$$
f_{n+1}\left(x_{1}, \ldots, x_{n}, x_{n+1}\right)=f_{2}\left(f_{n}\left(x_{1}, \ldots, x_{n}\right), x_{n+1}\right) .
$$

Starting from $n=2$ and applying (4) we get $f_{3}\left(x_{1}, x_{2}, x_{3}\right)=f_{2}\left(f_{2}\left(x_{1}, x_{2}\right), x_{3}\right)$, $f_{4}\left(x_{1}, x_{2}, x_{3}, x_{4}\right)=f_{2}\left(f_{3}\left(x_{1}, x_{2}, x_{3}\right), x_{4}\right)=f_{2}\left(f_{2}\left(f_{2}\left(x_{1}, x_{2}\right), x_{3}\right), x_{4}\right)$ and so on. Thus the whole aggregation rule $f$ is defined recursively by the binary aggregation rule $f_{2}$. From the definition of consistency in aggregation it follows that the binary rule has the following properties:

(Commutativity)

$$
f_{2}\left(x_{1}, x_{2}\right)=f_{2}\left(x_{2}, x_{1}\right)
$$

(Associativity)

$$
f_{2}\left(x_{1}, f_{2}\left(x_{2}, x_{3}\right)\right)=f_{2}\left(f_{2}\left(x_{1}, x_{2}\right), x_{3}\right) .
$$

Therefore $f_{2}$ is a commutative semigroup operation on $X$. Use of the multiplicative notation $f_{2}\left(x_{1}, x_{2}\right)=x_{1} x_{2}$ is therefore warranted. The recursive rule (4) shows that the aggregation rule is simply generated by repeated application of this operation. Conversely, let $x_{1} x_{2}$ be a commutative semigroup operation and define an aggregation rule using the equations (2) and (3). It is clear that an aggregation rule generated in this fashion is CIA.

As already mentioned in the introduction, the semigroup representation follows almost trivially from the definition of consistecy in aggregation. But as we also al- 
ready argued, this is exactly the point. The lack of a general definition has been the source of confusion surrounding the concept of consistency in aggregation. By adopting such a definition the algebraic structure of the property becomes self-evident. This is to our knowledge the first time such a general result has been presented. As we made no assumptions about the functional form or measurement scale of the aggregation rule, Theorem 1 represents a general statement of the implications of consistency in aggregation. Also, these implications are highly non-trivial. That an aggregation rule be thus reducible to repetition of a commutative semigroup operation is clearly a quite stringent requirement. However, it is not too stringent in the sense that only trivial aggregation rules would satisfy it. There exist many nontrivial commutative semigroup operations that have an aggregation interpretation in a variety of contexts. Furthermore, semigroup theory or more broadly abstract algebra is a well-developed theory and the existence of the semigroup representation shows that this theory is directly relevant to analysis of aggregation problems. We try to demonstrate the strength of this approach below by applying fundamental algebraic results to index number theory.

\section{Discussion and examples}

\subsection{Unweighted and weighted aggregation rules}

Perhaps the most fundamental aggregation method is aggregation of sets into larger sets. Let $A$ be a set and let $X \subset \mathcal{P}(A)$ be any collection of subsets of $A$ closed under finite unions (e.g. a Boolean algebra). The operation $B \cup C$ defined for any $B, C \in X$ is a commutative semigroup operation in $X$. It therefore defines a CIA aggregation rule. This rule is given by $f_{n}\left(B_{1}, \ldots, B_{n}\right)=\bigcup_{i=1}^{n} B_{i}$. Similarly it may be seen that the intersection operation $\cap$ defines a CIA aggregation rule in any class of subsets of $A$ closed under finite intersections.

Another fundamental aggregation method is addition. Adding real or complex numbers and vectors together is a commutative semigroup operation. The aggre- 
gation rule defined by addition is thus CIA. Also, multiplication of reals is trivially CIA, as are the aggregation rules defined by the operations $x y=\min \{x, y\}$ and $x y=\max \{x, y\}$. For example $x y=\min \{x, y\}$ defines the rule $f_{1}(x)=x$, $f_{n}\left(x_{1}, \ldots, x_{n}\right)=\min \left\{x_{1}, \ldots, x_{n}\right\}$.

What all of these basic examples have in common is that they are unweighted. There is no weighting variable giving the importance, for example population size, expenditure share, etc. of any of the measurements. In most cases of aggregation of economic data such auxiliary variables are necessarily present. However, our definition of CIA requires that the aggregation rule map the measurements into the same scale. This poses some difficulty, as is seen for example in Blackorby and Primont (1980), who derive strong results for the unweighted or one-variable case, but leave essentially open the case in which each measurement of interest is associated with one or more "attributes" to use their terminology. However, this problem can be easily overcome in the case of weights by including these explicitly in the aggregation rule. This is best shown by a simple example.

Consider the weighted arithmetic mean. It is always possible to calculate a grand mean as a mean of means. Thus it seems clear that the arithmetic mean should be considered CIA. However, the above mentioned complication involving the weights arises. Our definition of an aggregation rule requires that the aggregation has to result on an aggregate on the same scale as the original measurements are. We have two measurements on each statistical unit in our data, the variable of interest, say $x$ and the weight $\alpha$, but the arithmetic mean maps these to one real number. The solution is to define the aggregation method explicitly for the weights as well. For $\mathbf{x}=$ $(x, \alpha)$ and $\mathbf{y}=(y, \beta), \alpha, \beta>0$ define the following operation $\mathbf{x y}=\left(\frac{\alpha x+\beta y}{\alpha+\beta}, \alpha+\beta\right)$. The first component of the operation gives the weighted arithmetic mean of $x$ and $y$ and the second component simply adds the weights together. The operation is clearly commutative. Also, simple calculation will show that it is also associative. The arithmetic mean operation thus defines a CIA aggregation rule for real 2-vectors with strictly positive second component. It is precisely in this sense that the arithmetic mean may be considered CIA. 
It should be clear that this way of completing means to aggregation rules by adding weighting information may be used in many other contexts as well. The discussion of index numbers below will give an important example.

\subsection{Quasilinear means and aggregation rules}

A quasilinear aggregation rule is defined by a semigroup operation of the form $\mathbf{x y}=\mathbf{f}^{-1}[\mathbf{f}(\mathbf{x})+\mathbf{f}(\mathbf{y})]$, where $\mathbf{f}$ is a bijection from some subset of $\mathbb{R}^{K}$ (or $\mathbb{C}^{K}$ ) to another. Quasilinear aggregation rules are by definition always consistent in aggregation. In fact, it is a recurring result in various contexts that associativity axioms (such as consistency in aggregation) coupled with other regularity conditions imply a quasilinear form for aggregation rules defined for real numbers or vectors. Classical examples of such results are given in Nagumo (1930) and Kolmogorov (1930) concerning characterization of quasilinear means (see also Aczél (1966)). Quasilinearity results related to index numbers and thus relevant to the present study are given for example by Blackorby and Primont (1980), Blackorby and Donaldson (1984), Diewert (1993), Gorman (1986) and Balk (1996).

As mentioned, quasilinear means give a basic example of quasilinear aggregation. While means, quasilinear and other, are most often associated with aggregation of real numbers, we extend the conventional definition in an obvious way to cover aggregation of real vectors. This slight extension will prove to be useful in the next section on index number formulas. Consider a sequence $\mathbf{M}=\left(\mathbf{M}_{n}\right)_{n \in \mathbb{N}}$ of functions that produce some value $\overline{\mathbf{x}}^{\boldsymbol{\alpha}}=\mathbf{M}_{n}\left(\mathbf{x}_{1}, \ldots \mathbf{x}_{n} ; \boldsymbol{\alpha}\right)$ in an open set $A \subset \mathbb{R}_{++}^{K}$, for any finite collection of vectors $\mathbf{x}_{1}, \ldots, \mathbf{x}_{n} \in A$, that are measurements on some variable of interest and $\boldsymbol{\alpha}$ consisting of real numbers $\alpha_{1}, \ldots, \alpha_{n}>0$ that give the values of some weighting variable. The sequence $\mathbf{M}$ is a quasilinear weighted mean if there exists a continuous bijection $\mathbf{g}: A \rightarrow B$ to an open convex subset $B$ of $\mathbb{R}^{K}$ which satisfies

$$
\mathbf{M}_{n}\left(\mathbf{x}_{1}, \ldots, \mathbf{x}_{n} ; \boldsymbol{\alpha}\right)=\mathbf{g}^{-1}\left[\left(\sum_{j=1}^{n} \alpha_{j}\right)^{-1} \sum_{i=1}^{n} \alpha_{i} \mathbf{g}\left(\mathbf{x}_{i}\right)\right]
$$


Following the above example concerning the arithmetic mean, any quasilinear mean may be used to construct a $(K+1)$-dimensional semigroup operation of the form

$$
(\mathbf{x}, \alpha)(\mathbf{y}, \beta)=\left(\mathbf{g}^{-1}\left[\frac{\alpha \mathbf{g}(\mathbf{x})+\beta \mathbf{g}(\mathbf{y})}{\alpha+\beta}\right], \alpha+\beta\right) .
$$

This operation is clearly quasilinear and thus defines a quasilinear aggregation rule. Of course, this implies that any quasilinear mean is consistent in aggregation in the same sense that the arithmetic mean is. It is therefore natural to ask when the converse result is true, i.e. when does a general weighted mean admit a quasilinear mean representation. As mentioned, Nagumo (1930) and Kolmogorov (1930) give necessary and sufficient conditions for the one-variable case. In this section we use a technique based on the semigroup representation to derive a many-dimensional version of a Kolmogorov-Nagumo type result. This result will then be used to derive a quasilinear representation theorem for index number formulas. For reasons of simplicity and economic relevance we present the result for the set of strictly positive real vectors $\mathbb{R}_{++}^{K}$, but it should be clear that this result extends to other open subsets of $\mathbb{R}^{K}$ as well.

We start with a general definition of a weighted mean. Consider a sequence $\mathbf{M}=\left(\mathbf{M}_{n}\right)_{n \in \mathbb{N}}$ of functions $\mathbf{M}_{n}$ that produce some value $\overline{\mathbf{x}}^{\boldsymbol{\alpha}}=\mathbf{M}_{n}\left(\mathbf{x}_{1}, \ldots \mathbf{x}_{n} ; \boldsymbol{\alpha}\right) \in$ $\mathbb{R}_{++}^{K}$ for any finite collection of vectors $\mathbf{x}_{1}, \ldots, \mathbf{x}_{n} \in \mathbb{R}_{++}^{K}$ called measurements and real numbers $\alpha_{1}, \ldots, \alpha_{n}>0$ called weights. In the now familiar fashion, the sequence $\mathbf{M}$ may be completed to an aggregation rule $\mathbf{G}$ defined for $(K+1)$-vectors $\mathbf{a}_{i}=\left(\mathbf{x}_{i}, \alpha_{i}\right)$ by setting $\mathbf{G}_{n}\left(\mathbf{a}_{1}, \ldots, \mathbf{a}_{n}\right)=\left(\mathbf{M}_{n}(\mathbf{x}, \ldots \mathbf{x} ; \boldsymbol{\alpha}), \sum_{i=1}^{n} \alpha_{i}\right)$. The sequence $\mathbf{M}$ is said to be a weighted mean if $\mathbf{G}$ is consistent in aggregation as an aggregation rule. The corresponding semigroup $S$ is called a weighted mean semigroup. A weighted mean is said to be continuous if the function $\mathbf{M}_{n}$ is jointly continuous in the $\mathbf{x}_{i}$ and $\boldsymbol{\alpha}$ for any fixed $n$. The weighted mean is one-to-one if for fixed $\mathbf{x}_{-i}$ and $\boldsymbol{\alpha}$ it is a one-to-one map of $\mathbf{x}_{i}$. It is called homogeneous if $\mathbf{M}_{n}(\mathbf{x}, \ldots \mathbf{x} ; \boldsymbol{\alpha})=\mathbf{x}$ for any $\mathbf{x}$ and $\boldsymbol{\alpha}$. The next lemma describe some algebraic implications of these properties.

Lemma 1 The following statements are true for a weighted mean semigroup $S$ and 
any $\mathbf{a}=(\mathbf{x}, \alpha)$ in $S:$

1. $S$ is a topological semigroup iff the weighted mean is continuous.

2. $S$ is a cancellation semigroup iff the weighted mean is one-to-one.

3. Define the exponentiation operation by setting $\mathbf{a}^{\beta}=(\mathbf{x}, \alpha)^{\beta}=(\mathbf{x}, \alpha \beta)$ for any $\beta>0$. When $S$ has the continuity and homogeneity properties, $S$ is a cone semigroup with respect to this operation.

Proof. Claim 11 is self-evident. For claim 2 assume that the one-to-one condition holds. Then let $\mathbf{a b}=\mathbf{a} \mathbf{b}^{\prime}$ for some $\mathbf{a}=(\mathbf{x}, \alpha), \mathbf{b}=(\mathbf{y}, \boldsymbol{\beta})$ and $\mathbf{b}^{\prime}=\left(\mathbf{y}^{\prime}, \boldsymbol{\beta}^{\prime}\right)$ in S.Aggregation of weights implies that we must have $\beta=\beta^{\prime}$. But then by the one-to-one condition we must also have $\mathbf{y}=\mathbf{y}^{\prime}$ and therefore $\mathbf{b}=\mathbf{b}^{\prime}$ so that $S$ is a cancellation semigroup. To see the converse result, assume that $S$ is a cancellation semigroup but that the corresponding weighted mean is not one-to-one. Then, for some $\mathbf{a}_{i}=\left(\mathbf{x}_{i}, \alpha_{i}\right), i=1, \ldots, n$ and $\mathbf{a}_{1}^{\prime}=\left(\mathbf{x}_{1}^{\prime}, \alpha_{1}\right)$ we have $\mathbf{M}_{n}\left(\mathbf{x}_{1}, \ldots \mathbf{x}_{n} ; \boldsymbol{\alpha}\right)=$ $\mathbf{M}_{n}\left(\mathbf{x}_{1}^{\prime}, \ldots \mathbf{x}_{n} ; \boldsymbol{\alpha}\right)$. But then $\mathbf{a}_{1} \cdots \mathbf{a}_{n}=\mathbf{a}_{1}^{\prime} \cdots \mathbf{a}_{n}$ which violates the cancellativity assumption.

To see that claim 3 is true, note that by the homogeneity condition we have $\mathbf{a}^{n}=(\mathbf{x}, \alpha) \cdots(\mathbf{x}, \alpha)=(\mathbf{x}, n \alpha)$ so that the operation $\mathbf{a}^{\beta}=(\mathbf{x}, \alpha)^{\beta}=(\mathbf{x}, \alpha \beta)$ always coincides with exponentiation by a positive natural number in $S$. Also, the exponentiation operation is clearly jointly continuous in $(\mathbf{a}, \beta)$. Property (a) of a cone semigroup defined in Section 2 follows directly from the definition of $\mathbf{a}^{\beta}$. Property (c) is implied by the homogeneity property. This leaves property (b). Commutativity and associativity imply that for any natural number $n$ we have $(\mathbf{a b})^{n}=\mathbf{a}^{n} \mathbf{b}^{n}$. Also, if $m$ is a natural number, clearly $\left(\mathbf{a}^{\frac{1}{m}} \mathbf{b}^{\frac{1}{m}}\right)^{m}=\mathbf{a b}$ so that $(\mathbf{a b})^{\frac{1}{m}}=\mathbf{a}^{\frac{1}{m}} \mathbf{b}^{\frac{1}{m}}$. Therefore, for any natural $n, m,(\mathbf{a b})^{\frac{n}{m}}=\left[(\mathbf{a b})^{n}\right]^{\frac{1}{m}}=\left(\mathbf{a}^{n} \mathbf{b}^{n}\right)^{\frac{1}{m}}=\mathbf{a}^{\frac{n}{m}} \mathbf{b}^{\frac{n}{m}}$. As the exponentiation operation is continuous, this implies that (ii) holds for any positive real number.

A basic problem in algebra concerns topological embedding of semigroups into groups, for example Rothman (1960) and Lawson (1990) discuss such embeddings. 
A particular case of this problem is the embedding of cone semigroups into linear spaces, discussed for example in Lawson and Madison (1971). We now prove a quasilinear representation theorem for multi-dimensional weighted means based on a linear space embedding of the weighted mean semigroup ${ }^{1}$.

Theorem $\mathbf{2}$ A weighted mean $\mathbf{M}$ is quasilinear iff it is (i) continuous, (ii) monotone, and (iii) homogeneous.

Proof. It is clear that any quasilinear mean is a weighted mean and satisfies (i)-(iii). To see the converse let $S$ be a weighted mean semigroup on $\mathbb{R}_{++}^{K+1}$ of a mean satisfying (i)-(iii). By Lemma $1 S$ is a topological cancellation semigroup with positive exponentiation.

It is well known that any commutative cancellation semigroup may be embedded in a commutative group $G$ (for details, see Grillet (2001)). This means that there exists a group $G$ along with a one-to-one homomorphism $p: S \rightarrow G$. Thus, the semigroup $S$ and the subsemigroup $S^{\prime}=p(S)$ of $G$ are isomorphic as semigroups. For completeness, we give a sketch of how this may be done. The product set $S \times S$ with the coordinatewise operation is clearly a semigroup. Define a relation $R$ in $S \times S$ by $(\mathbf{a}, \mathbf{b}) R\left(\mathbf{a}^{\prime}, \mathbf{b}^{\prime}\right)$ iff $\mathbf{a b}^{\prime}=\mathbf{a}^{\prime} \mathbf{b}$. It is easy to check that this is a congruence, that the quotient semigroup $G=S \times S \mid R$ is in fact a group and that the map $p$ mapping each $\mathbf{a}$ in $S$ to the equivalence class of $\left(\mathbf{a}^{2}, \mathbf{a}\right)$ is a semigroup isomorphism. Also, as $S$ is defined on an open subset $\mathbb{R}_{++}^{K+1}$ of an Euclidean space, by Propositions 2.4 and 2.5 (i) in Lawson (1990) the embedding is also topological in the following sense: (i) the group $G$ is a topological group with respect to the relevant quotient topology of the product topology of $S \times S$ with $S$ open in $G$ and (ii) the function $p$ is continuous and open. Thus $S$ is identical with $S^{\prime}$ topologically as well as algebraically, and therefore we will equate the two from now on.

It is again well-known (see e.g. Rådström (1952)) that we may extend the positive exponentiation operation to exponentiation any real number on $G$. To see this, note

\footnotetext{
${ }^{1}$ Another way of looking at this is to regard it as a special case of the general results concerning the associativity functional equation given in Aczél and Hosszú (1956) and the Theorem of Section 8.2.4 in Aczél (1966).
} 
that it follows from construction of $G$ that each element $\mathbf{a}$ of $G$ has a representation $\mathbf{a}=\mathbf{u} \mathbf{v}^{-1}$ with $\mathbf{u}, \mathbf{v}$ in $S$ and where $\mathbf{v}^{-1}$ denotes the inverse element of $\mathbf{v}$ in $G$. For any $\mathbf{a}=\mathbf{u v}^{-1}$ in $G$ define $\mathbf{a}^{\beta}=\mathbf{u}^{\beta}\left(\mathbf{v}^{\beta}\right)^{-1}$ for $\beta>0$, then define $\mathbf{y}^{0}=\mathbf{e}$, where $\mathbf{e}$ is the identity element of $G$ and finally $\mathbf{a}^{\beta}=\left(\mathbf{a}^{-1}\right)^{-\beta}$ for $c<0$. That this is well-defined, agrees with positive exponentiation on $S$ and satisfies conditions (a),(b) and (c) is easy to check by considering the various cases corresponding to the different signs of the exponents. As an example, let $\beta>0, \gamma<0, \beta+\gamma>0$ and $\mathbf{a}=\mathbf{u v}^{-1}$. Then $\mathbf{a}^{\beta} \mathbf{a}^{\gamma}=\mathbf{a}^{\beta} \mathbf{a}^{\gamma}(\mathbf{u v})^{\beta+\gamma}\left[(\mathbf{u v})^{\beta+\gamma}\right]^{-1}=\mathbf{u}^{2 \beta+\gamma} \mathbf{v}^{\beta}\left(\mathbf{u}^{\beta} \mathbf{v}^{2 \beta+\gamma}\right)^{-1}=\mathbf{u}^{\beta+\gamma}\left(\mathbf{v}^{\beta+\gamma}\right)^{-1}=$. $\mathbf{a}^{\beta+\gamma}$.

The extension of exponentiation makes $G$ a linear space with the group operation as vector addition and exponentiation as scalar multiplication. It is clear that the semigroup $S$ is an open convex cone in $G$, i.e. the function $p$ embeds $S$ algebraically and topologically as a convex cone in the linear space $G$. Let now $\mathbf{h}: \mathbb{R}^{L} \rightarrow G$ be any linear map $\mathbf{h}(\boldsymbol{\lambda})=\mathbf{u}_{1}^{\lambda_{1}} \cdots \mathbf{u}_{L}^{\lambda_{L}}$ with $\mathbf{u}_{i}$ in the subsemigroup $S$. Clearly, $\mathbf{h}$ is continuous on $\mathbb{R}_{++}^{L}$ and thus on all $\mathbb{R}^{L}$. It is therefore a straightforward implication of the invariance of domain theorem that the dimension of $G$ as a linear space must be $K+1$. Let $U$ consisting of vectors $\mathbf{u}_{1}, \cdots, \mathbf{u}_{K+1}$ be a basis of $G$ and let $\mathbf{t}: G \rightarrow \mathbb{R}^{K+1}$ be the linear map mapping any $\mathbf{a}$ in $G$ to its $U$-coordinates. As it is linear, $\mathbf{t}$ is in particular additive so that $\mathbf{t}[(\mathbf{x}, \alpha)(\mathbf{y}, \beta)]=\mathbf{t}(\mathbf{x}, \alpha)+\mathbf{t}(\mathbf{y}, \beta)$ for $(\mathbf{x}, \alpha),(\mathbf{y}, \beta)$ in $S$. Also, by linearity $\mathbf{t}$ is positively linear homogeneous so that $\mathbf{t}(\mathbf{x}, \alpha)=\alpha \mathbf{t}(\mathbf{x}, 1)$. This implies that the function $\mathbf{g}$ defined for $\mathbf{x}$ in $S$ by $\mathbf{g}(\mathbf{x})=\mathbf{t}(\mathbf{x}, 1)$ gives a quasilinear representation of $\mathbf{M}$.

\subsection{Some remarks on the generality of CIA}

As most of the present study concerns aggregation of real numbers and vectors, it is perhaps necessary to discuss briefly the generality of the notion of consistency in aggregation. There is no need in principle to restrict attention to real (or complex) vectors, while these of course provide the most immediate examples. The above discussion of unions and intersections shows that CIA as a concept is perfectly applicable 
to aggregation of sets. It may similarly, be applied to aggregation of functions or relations. To give a simple example, there is a natural way to derive aggregation rules for functions that map into a semigroup. Let $S$ be a commutative semigroup and let $\mathcal{F}(A, S)$ be the set of functions $A \rightarrow S$. We may always define a semigroup in $\mathcal{F}(A, S)$ by pointwise application of the operation of $S$. For any $f, g \in \mathcal{F}(A, S)$ define the operation $f g$ with $f g(a)=f(a) g(a)$ for any $a \in A$. This operation may be then used to construct an aggregation rule satisfying CIA for functions $A \rightarrow S$. Of course, we need not be interested in the whole set $\mathcal{F}(A, S)$, but some subset of it given by additional structure on $A$ which is preserved by the semigroup operation on $S$.

For example, if $S$ is topological and there is a topology on $A$ the set of continuous functions $A \rightarrow S$ is clearly a semigroup and may again be used to define an aggregation rule for continuous functions. Similarly, given the necessary structure, we may define a subsemigroup of $\mathcal{F}(A, S)$ consisting of random variables from $A$ interpreted as a sample space to $S$ and use this to construct an aggregation rule for random variables. An example of such an aggregation rule for functions is given by the convolution operation on the set of Lebesgue integrable functions in $\mathbb{R}^{n}$. It is well known that this is a commutative semigroup operation and therefore defines an aggregation rule for integrable functions which is consistent in aggregation. It is also well known that the convolution semigroup is isomorphic via the Fourier transform to a subsemigroup of $\mathcal{F}\left(\mathbb{R}^{n}, \mathbb{C}\right)$ with $\mathbb{C}$ interpreted as a multiplicative semigroup. Still another example is given by the one-dimensional weighted mean semigroups defined in the previous section. Using the pointwise definition for the first component, we may use any weighted mean to define a semigroup operation for pairs $(u, \alpha)$ where $u$ is a real-valued function and $\alpha$ is a strictly positive weight. If we interpret $u$ as an utility function on some set $A$ and $\alpha$ as a population size, the operation gives an aggregation rule for utility functions. This may be interpreted as calculation of social welfare for variable-sized populations (see Blackorby and Donaldson (1984) for discussion of such rules).

While space limitations do not permit anything resembling a full treatment of 
such topics, we hope that the examples go some way of convincing the reader that CIA as a concept and the underlying semigroup structure are general enough to permit analysis of a wide variety of different aggregation problems.

\section{Index numbers and consistency in aggregation}

\subsection{The index number problem}

We start with a brief outline of the index number problem. Price and quantity measurements on $n$ commodities have been collected on two periods, $t=0,1$, called the base and comparison periods respectively. Let $\mathbf{p}^{t}=\left(p_{1}^{t}, \ldots, p_{n}^{t}\right), t \in\{0,1\}$, $p_{i}^{t}>0$ denote the prices of the two periods and $\mathbf{q}^{t}=\left(q_{1}^{t}, \ldots, q_{n}^{t}\right), t=0,1, q_{i}^{t}>0$ the quantities. Let $\pi_{i}=\frac{p_{i}^{1}}{p_{i}^{0}}$ and $\kappa_{i}=\frac{q_{i}^{1}}{q_{i}^{0}}$ be the price and quantity relatives associated with the measurements and let $v_{i}^{t}=p_{i}^{t} q_{i}^{t}$ be the value or expenditure associated with each commodity on period $t$. Thus, each commodity is associated with vectors $\boldsymbol{\pi}_{i}=\left(\pi_{i}, v_{i}^{0}, v_{i}^{1}\right)$ and $\boldsymbol{\kappa}_{i}=\left(\kappa_{i}, v_{i}^{0}, v_{i}^{1}\right)$. The aggregate value for period $t$ is denoted $V^{t}=\sum_{i=1}^{n} v_{i}^{t}$.

For one commodity, the value ratio has a canonical, uniquely determined multiplicative decomposition $\frac{v^{1}}{v^{0}}=\pi \kappa$ into the price and quantity relatives. The index number problem concerns aggregation of such commodity-level decompositions into a decomposition $\frac{V^{1}}{V^{0}}=P Q$, where $P$ is a price index describing the aggregate change in the "price level" and $Q$ is a quantity index describing aggregate change in quantities consumed or produced. Price and quantity indices are simply proposed solutions to the aggregate decomposition problem. A price index formula is a rule mapping all possible price-quantity situations to a price index, while a quantity index formula is defined analogously. Note that the decomposition formulation implies that only either a price index formula or a quantity index formula needs to be chosen, as the other indicator is then defined as a multiplicative residual. For example, if we have decided that the value for the price index should be $P$, then the value of the quantity index $Q$ must be $Q=\frac{V^{1}}{V^{0} P}$. A price index formula and a quantity index formula 
which always give a multiplicative decomposition of the aggregate value ratio constitute what is usually called an index number pair. Because either index describes the index number pair completely, the following discussion is formulated entirely in terms of price indices. For quantity indices, a completely identical argument could be presented, simply by replacing the vectors $\boldsymbol{\pi}_{i}$ with the vectors $\boldsymbol{\kappa}_{i}$.

As mentioned, a price index formula maps all possible price-quantity combinations to a price index. Formally this implies that a price index formula must be a sequence of functions, each giving the formula for $n$ observations. Following convention (see Vartia (1976), Gorman (1986), Balk (1996)), we require that a price index formula depend on the prices and quantities via the vectors $\boldsymbol{\pi}_{i}=\left(\pi_{i}, v_{i}^{0}, v_{i}^{1}\right)$ alone. Thus, we define a price index formula $g$ to be a sequence $\left(g_{n}\right)_{n \in \mathbb{N}}$ of functions $g_{n}: \mathbb{R}_{++}^{3 n} \rightarrow \mathbb{R}_{++}$so that the price index $P$ for any price-quantity measurements is given by $P=g_{n}\left(\boldsymbol{\pi}_{1}, \ldots, \boldsymbol{\pi}_{n}\right)$ and the index number pair corresponding to the same measurements is given by $(P, Q)=\left(P, \frac{V^{1}}{V^{0} P}\right)$.

\subsection{Index number semigroups}

Consistency in aggregation for index number formulas is formulated using the by now familiar technique of incorporating weights into the aggregation rule. A price index formula aggregates measurements consisting of vectors $\left(\pi_{i}, v_{i}^{0}, v_{i}^{1}\right)$ to a positive real number which may be loosely interpreted as "an aggregate price relative". In other words the price index is an aggregate corresponding to the first components of the measurements, the price relative. An aggregation rule is obtained from the price index formula by taking aggregation of the values $v_{i}^{0}$ and $v_{i}^{1}$ explicitly into consideration. The values are of course aggregated simply by adding them together. Any price index formula $g$ therefore generates an aggregation rule $\mathbf{G}$ consisting of vector-valued functions $\mathbf{G}_{n}$ given by

$$
\mathbf{G}_{n}\left(\boldsymbol{\pi}_{1}, \ldots, \boldsymbol{\pi}_{n}\right)=\left(g_{n}\left(\boldsymbol{\pi}_{1}, \ldots, \boldsymbol{\pi}_{n}\right), \sum_{i=1}^{n} v_{i}^{0}, \sum_{i=1}^{n} v_{i}^{1}\right) .
$$


An index number formula $g$ is said to be consistent in aggregation if the corresponding aggregation rule $\mathbf{G}$ is consistent in aggregation in the sense of the general definition. We call the semigroup $S$ defined by a price index exhibiting CIA a price index semigroup.

A price index formula and the corresponding semigroup are said to be monotone $e^{2}$ if for any $\boldsymbol{\pi}_{1}, \boldsymbol{\pi}_{2}$ in $S$ the operation $\boldsymbol{\pi}_{1} \boldsymbol{\pi}_{2}$ is strictly monotone in $\pi_{1}$ (and $\pi_{2}$ ). A price index formula and the corresponding semigroup are weakly proportional if $\left(\pi, \alpha v_{1}^{0}, \alpha v_{1}^{1}\right)\left(\pi, \beta v_{1}^{0}, \beta v_{1}^{1}\right)=\left(\pi,(\alpha+\beta) v_{1}^{0},(\alpha+\beta) v_{1}^{1}\right)$. It is straightforward to see that this coincides with the condition that if both the prices and quantities of all commodities have changed proportionally then the price index should be equal to the common price relative.

The representation of price indices as functions of $\boldsymbol{\pi}_{i}=\left(\pi_{i}, v_{i}^{0}, v_{i}^{1}\right)$ is conventional. However, another representation is also useful for our purposes. Consider the function $\mathbf{m}$ defined by $\mathbf{m}\left(\pi, v^{0}, v^{1}\right)=\left(\pi, \frac{v^{1}}{v^{0} \pi}, v^{0}\right)$, which maps any vector $\boldsymbol{\pi}=\left(\pi, v^{0}, v^{1}\right)$ bijectively to the vector $\boldsymbol{\eta}=\mathbf{m}(\boldsymbol{\pi})=\left(\pi, \kappa, v^{0}\right)$. We may therefore define a new semigroup operation $\boldsymbol{\eta}_{1} \circ \boldsymbol{\eta}_{2}=\mathbf{m}\left[\mathbf{m}^{-1}\left(\boldsymbol{\eta}_{1}\right) \mathbf{m}^{-1}\left(\boldsymbol{\eta}_{2}\right)\right]$ for the transformed measurements. Call this semigroup $\bar{S}$. It is isomorphic to the index number semigroup $S$ as the function $\mathbf{m}: \bar{S} \rightarrow S$ is an isomorphism. The transformed semigroup operation defines a transformed aggregation rule. The basic properties of the semigroup $\bar{S}$ are given in the next lemma.

Lemma 2 Let $S$ be an index number semigroup and let $\bar{S}$ be the isomorphic semigroup defined above. Let $\boldsymbol{\eta}_{i}=\mathbf{m}\left(\boldsymbol{\pi}_{i}\right)=\left(\pi_{i}, \kappa_{i}, v_{i}^{0}\right) \in \bar{S}$ for $i=1, \ldots, n$ and let $\left(P, V^{0}, V^{1}\right)=\boldsymbol{\pi}_{1} \cdots \boldsymbol{\pi}_{n}$. Furthermore, let $Q$ be the quantity index corresponding to the price index $P$, so that $P Q=\frac{V^{1}}{V^{0}}$. Then $\left(P, Q, V^{0}\right)=\boldsymbol{\eta}_{1} \circ \cdots \circ \boldsymbol{\eta}_{n}$.

Proof. Let $(\widetilde{P}, \widetilde{Q}, \widetilde{V})=\boldsymbol{\eta}_{1} \circ \cdots \circ \boldsymbol{\eta}_{n}$. Then it follows from the definition of $\bar{S}$ that $\mathbf{m}(\widetilde{P}, \widetilde{Q}, \widetilde{V})=\left(P, V^{0}, V^{1}\right)$. Taking $\mathbf{m}^{-1}$ on both sides the result follows.

\footnotetext{
${ }^{2}$ Note that this monotonicity condition is different from the axiom requiring monotonicity in comparison period prices. The problem with monotonicity axioms in general is that monotonicity in the variables of some representation may not translate into anything intuitive in some other representation.
} 
Recalling our definition of weighted means in Section 5.2 it is clear that the semigroup $\bar{S}$ gives a representation of the index number pair as a $v_{i}^{0}$-weighted 2dimensional mean of the $\left(\pi_{i}, \kappa_{i}\right)$. The lemma thus implies that consistency in aggregation for an index number formula is equivalent to the requirement that the corresponding index number pair is a two-dimensional base period value-weighted mean of the price relative-quantity relative pairs. Given our characterization of quasilinear means, it is clear that under natural regularity conditions, the index number pair must in fact be a quasilinear mean of the price and quantity relatives. The result is given in the following theorem. The quasilinearity part of the theorem, which is simply a special case of Theorem 2, may be seen as an extension of Blackorby and Primont (1980) quasilinearity theorem for one variable. Gorman (1986) derives similar results, albeit using a very different technique and stronger assumptions. As may be seen in the proof, the semigroup structure reduces the problem to a series of classical algebraic embedding problems, so that we do not need to introduce the various separability concepts used by Gorman. Balk (1996) uses a quasilinear form as definition of consistency in aggregation. Our result implies that under additional conditions our general concept of consistency in aggregation reduces to Balk's narrower definition in the case of index number formulas ${ }^{3}$.

Theorem 3 A price index formula is consistent in aggregation if and only if the index number pair $(P, Q)=\left(P, \frac{V^{1}}{V^{0} P}\right)$ can be represented as a $v_{i}^{0}$-weighted mean of $\left(\pi_{i}, \kappa_{i}\right)$. This mean is quasilinear iff the price index number formula is continuous, monotone and weakly proportional as well as consistent in aggregation.

Proof. The first part was argued in the above paragraph. To show the second part we must show that continuity, monotonicity and weak proportionality are equivalent to the conditions (i)-(iii) of Theorem 2. Let $S$ be the index number semigroup defined by $P$ and let $\bar{S}$ be the corresponding weighted mean semigroup. $\bar{S}$ is a topological semigroup iff $S$ is a topological semigroup, as $\mathbf{m}$ and it inverse

\footnotetext{
${ }^{3}$ The example $g_{n}\left(\boldsymbol{\pi}_{1}, \ldots, \boldsymbol{\pi}_{n}\right)=\min \left\{\pi_{1}, \ldots, \pi_{n}\right\}$ shows that an index number formula may be CIA without being quasilinear.
} 
given by $\mathbf{m}^{-1}\left(\pi, \kappa, v^{0}\right)=\left(\pi, v^{0}, \pi \kappa v^{0}\right)$ are continuous. Also, it is clear that $\bar{S}$ is a topological semigroup iff the index number formula is continuous. Continuity of the index number formula is therefore equivalent to property (i) in Theorem 2.Also, as an isomorphic semigroup $\bar{S}$ is cancellative if and only if $S$ is cancellative. This implies that monotonicity of the index number formula and property (ii) in Theorem 2 are equivalent. Finally, note that for $\boldsymbol{\eta}=\left(\pi, \kappa, v^{0}\right)$ and $\widetilde{\boldsymbol{\eta}}=\left(\pi, \kappa, \widetilde{v}^{0}\right)$ we have $\mathbf{m}^{-1}(\boldsymbol{\eta})=\left(\pi, v^{0}, \pi \kappa v^{0}\right)$ and $\mathbf{m}^{-1}(\widetilde{\boldsymbol{\eta}})=\left(\pi, \widetilde{v}^{0}, \pi \kappa \widetilde{v}^{0}\right)$, so that weak proportionality of $S$ implies that $\mathbf{m}^{-1}(\boldsymbol{\eta}) \mathbf{m}^{-1}(\widetilde{\boldsymbol{\eta}})=\left(\pi,\left(v^{0}+\widetilde{v}^{0}\right) \cdot 1,\left(v^{0}+\widetilde{v}^{0}\right) \pi \kappa\right)$. But then $\boldsymbol{\eta} \circ \widetilde{\boldsymbol{\eta}}=\left(\pi, \kappa, v^{0}+\widetilde{v}^{0}\right)$ so that $\bar{S}$ is homogeneous. Similarly, for $\boldsymbol{\pi}=\left(\pi, \alpha v_{1}^{0}, \alpha v_{1}^{1}\right)$ and $\widetilde{\boldsymbol{\pi}}=\left(\pi, \beta v_{1}^{0}, \beta v_{1}^{1}\right)$ we have $\mathbf{m}(\boldsymbol{\pi})=\left(\pi, \frac{v^{1}}{v^{0} \pi}, \alpha v^{0}\right)$ and $\mathbf{m}(\widetilde{\boldsymbol{\pi}})=\left(\pi, \frac{v^{1}}{v^{0} \pi}, \beta v^{0}\right)$. Then if $\bar{S}$ satisfies the homogeneity condition $\mathbf{m}(\boldsymbol{\pi}) \circ \mathbf{m}(\tilde{\boldsymbol{\pi}})=\left(\pi, \frac{v^{1}}{v^{0} \pi},(\alpha+\beta) v^{0}\right)$. But then $\boldsymbol{\pi} \tilde{\boldsymbol{\pi}}=\left(\pi,(\alpha+\beta) v^{0},(\alpha+\beta) v^{1}\right)$ so that $S$ is weakly proportional.

To show the relation between standard representations of index numbers and ours we consider a few well-known formulas. Any index number pair that satisfies the conditions of the previous theorem has a representation ${ }^{4}$ of the form

$$
(P, Q)=\mathbf{g}^{-1}\left[\left(V^{0}\right)^{-1} \sum_{i=1}^{n} v_{i}^{0} \mathbf{g}\left(\pi_{i}, \kappa_{i}\right)\right]
$$

The most basic example is the Laspeyres price index $P_{L}$ which corresponds to the semigroup operation $\boldsymbol{\pi}_{1} \boldsymbol{\pi}_{2}=\left(\frac{v_{1}^{0} \pi_{1}+v_{2}^{0} \pi_{2}}{v^{1}+v^{0}}, v_{1}^{0}+v_{2}^{0}, v_{1}^{1}+v_{2}^{1}\right)$. It is well-known that the Laspeyres price index and the Paasche quantity index $Q_{P}$ form an index number pair. As the Laspeyres price index clearly satisfies the conditions for quasilinearity, the Laspeyres-Paasche pair has a representation of the above form. In fact, replacing the function $\mathbf{g}$ in the equation with $\mathbf{g}_{L}(\pi, \kappa)=(\pi-1, \pi(\kappa-1))$ gives the Laspeyres-Paasche pair. The Paasche-Laspeyres pair is given by switching the roles of quantities and prices, that is, choosing $\mathbf{g}_{P}(\pi, \kappa)=(\kappa(\pi-1), \kappa-1)$. The Stuvel (1957) price-quantity index pair is given by the average of these two, or

\footnotetext{
${ }^{4}$ Note that while any index number formula pair with the required properties may be represented as a two-dimensional quasilinear mean not all two-dimensional means define an index number pair. This is because of the condition that $P Q=\frac{V^{1}}{V^{0}}$.
} 
$\mathbf{g}_{S}(\pi, \kappa)=\frac{1}{2}\left[\mathbf{g}_{L}(\pi, \kappa)+\mathbf{g}_{P}(\pi, \kappa)\right]$. The quasilinear mean representation for the Montgomery-Vartia price-quantity index pair (Vartia (1976)) is given by $\mathbf{g}_{V}(\pi, \kappa)=$ $l(\pi \kappa)(\log \pi, \log \kappa)$ where $l(\pi k)=\frac{\pi \kappa-1}{\log \pi k}$ when $\pi \kappa \neq 1$ and $l(1)=1$.

\section{References}

ACZÉL, J - HOSSZÙ, M. (1956): On Transformations with Several Variables and Operations in Multidimensional Spaces. Acta Mathematica Acad. Sci. Hungaricae, 7, 327-338.

ACZÉL, J. (1966): Lectures on Functional Equations and their Applications. Academic Press, New York.

BALK, B. M. (1996): Consistency-in-Aggregation and Stuvel Indices. Review of Income and Wealth 42: 353-363.

BLACKORBY, C., DONALDSON, D. (1984): Social Criteria for Evaluating Population Change. Journal of Public Economics 25: 13-33.

BLACKORBY, C., PRIMONT, D., RUSSELL, R.R. (1978): Duality, Separability, and Functional Structure: Theory and Economic Applications. North Holland, New York.

BLACKORBY, C., PRIMONT, D. (1980): Index Numbers and Consistency in Aggregation. Journal of Economic Theory 22: 87-98.

DIEWERT, W. E. (1978): Superlative Index Numbers and Consistency in Aggregation. Econometrica, 46: 883-900.

- (1993): Essays on Index Numbers. North Holland.

GORMAN, W.M.(1986): Compatible Indices. Economic Journal, Supplement: Conference papers 96: 83-95.

GRILLET, P. A. (2001): Commutative Semigroups. Kluwer Academic Publishers. Dordrecht.

KOLMOGOROV, A.I.(1930): Sur la notion de la moyenne. Atti della R. Accademia Nazionale dei Lincei 12, 388-391.

LAWSON, J., MADISON, B. (1971): On Congruences and Cones. Mathematische Zeitschrift, 120, $18-24$.

LAWSON, J. (1990): Embedding Semigroups in Lie Groups. In Hoffman, L. Hoffman, P. and Hoffman (eds.), K:Analytical and Topological Theory of Semigroups: Trends and Developments. Walter de Gruyter.

NAGUMO (1930): Über eine Klasse der Mittelwerde. Japanese Journal of Mathematics 7: 71-79.

PURSIAINEN, H. (2005): Consistent Aggregation Methods and Index Number Theory. A PhD dissertation, Univ. of Helsinki.

RÅdSTRÖM, H. (1952): An Embedding Theorem for Spaces of Convex Sets, Proc. Am. Math. Soc. 3: $165-169$.

ROTHMAN, N. J. (1960): Embedding of Topological Semigroups. Math. Annalen 139: 197-203.

STUVEL, G. (1957): A New Index Number Formula. Econometrica 25, 123-131.

- (1989): The Index Number Problem and Its Solution. Macmillan Press Ltd., London.

VARTIA, Y. O. (1976): Relative Changes and Index Numbers. Research Institute for the Finnish Economy, Series A. 